\title{
Manfred Messerschmidt Aufhebung des Todesurteils gegen Franz Jägerstätter
}

Der 2. Senat des Reichskriegsgerichts (RKG) verurteilte den 36jährigen Bauern Franz Jagerstätter aus St. Radegund, Vater von drei Kindern im Alter von drei bis sechs Jahren, am 6. Juli 1943 zum Tode. Für Soldaten, die aus religiösen und Gewissensgründen den Wehrdienst generell oder jedenfalls den Dienst mit der Waffe verweigerten, sah die bei Kriegsbeginn in Kraft gesetzte Kriegssonderstrafrechtsverordnung (KSSVO) die Todesstrafe vor. Eine unmittelbar dem Wortlaut der Verordnung zu entnehmende Regelung war dies aber nicht, vielmehr ist auf Wehrdienstverweigerer die Generalklausel des $₫$ s Abs. I Ziff. 3 KSSVO angewendet worden, die alle nicht speziell geregelten Fälle der Wehrdienstentziehung erfassen sollte, und zwar mit der Formulierung »wer es unternimmt, sich ... auf andere Weise der Erfüllung des Wehrdienstes ganz, teilweise oder zeitweise zu entziehen«, wird wegen Zersetzung der Wehrkraft mit dem Tode bestraft.

Die 17. Strafkammer des Landgerichts Berlin hat das Todesurteil mit Beschluß vom 7. Mai 1997 aufgehoben, weil es auf politischen Gründen beruht hat. Sie hat überdies formale Gründe für diese Entscheidung herangezogen: Die KSSVO ist vom Alliierten Kontrollrat am 30. Januar 1946 zusammen mit anderen nationalsozialistischen $\mathrm{Ge}$ setzen aufgehoben worden. Auf derartige Vorschriften bezieht sich $\$ I Abs. I des Gesetzes zur Wiedergutmachung nationalsozialistischen Unrechts auf dem Gebiet des Strafrechts vom 5. Januar 195 I (NS-StrWG) mit der Anordnung, daß gerichtliche Entscheidungen in der Zeit vom 30. Januar 1933 bis zum 8. Mai 1945 aufzuheben sind, wenn sie auf Vorschriften beruhen, die die Festigung des Nationalsozialismus bezweckten, oder wenn die Entscheidungen aus politischen, rassischen oder religiösen Gründen ergangen sind.

Das Landgericht geht unter Aufgabe seiner bisherigen Rechtsprechung davon aus, daß das RKG-Urteil gegen Jägerstätter »in den Anwendungsbereich des $₫$ I Abs. I NS-StrWG fällt«. Es schließt eine Verurteilung aus politischen und religiösen Gründen daraus, daß der 2. Senat des RKG die Feststellung für bedeutsam erachtet hat, daß der Angeklagte weder Mitglied der NSDAP noch einer ihrer Gliederungen war und daß er ein Gegner des Nationalsozialismus sei. Politische Gründe charakterisieren das Urteil insbesondere, weil mit dem Todesurteil »die politisch gewollte Abschreckung « erreicht werden sollte. Besondere Beachtung verdient die Feststellung des Landgerichts, daß heutige Rechtsmaßstäbe zugrunde zu legen seien, wonach die Entscheidung, aus Gewissensgründen keinen Wehrdienst mit der Waffe zu leisten, zu respektieren sei.

Das Landgericht hätte weitere wichtige politische Gesichtspunkte berücksichtigen können, die seine Feststellung, das RKG habe sich im Gegensatz zu Urteilen des Volksgerichtshofes um eine formaljuristisch korrekte Begründung bemüht, in einem anderen Licht erscheinen lassen. So hat das RKG sogenannte "Rechtsgrundsätze" formuliert, in denen gesagt war, daß gegen den »hartnäckigen Überzeugungstäter « wegen der propagandistischen Wirkung seines Verhaltens »im Normalfall die Todesstrafe angezeigt« sei. ${ }^{1}$ Diese »Grundsätze « haben dazu geführt, daß Urteile zustande kamen, bei denen man sich keine Mühe machte zu prüfen, ob Milderungsgründe gegeben waren. Viele Urteile sind wie im Telegrammstil formuliert, mit Urteilsgründen von nicht mehr als einer Schreibmaschinenseite. Der 3. Senat des RKG

I Gesetzesdienst fur die Wehrmachtgerıchte, OKW/WR, 1941, Bundesarchıv-Militararchıv (BA-MA) Freiburg, RW 2/v. ${ }_{23}$ D, S. 5-1s. 
hatte schon im Mai 1940 mit typischer NS-Rechtsvorstellung entschieden, die Frage eines minder schweren Falles könne nicht von der Person des Täters aus, sondern vom Standpunkt der Allgemeinheit und von den Kriegsnotwendigkeiten her beurteilt werden. Entsprechend interpretierte die dem Reichskriegsgericht zugeordnete Reichskriegsanwaltschaft die NS-Gemeinschaftsethik: »Zersetzung der Wehrkraft ist die Störung oder Beeinträchtigung der totalen völkischen Einsatzbereitschaft zur Erringung des Endsieges in diesem Krieg «. ${ }^{2}$ Der von der Militärjustiz meistbenutzte Kommentar zum Militärstrafgesetzbuch und zur KSSVO umschrieb den politischen Charakter des $\mathbb{5} 5$ KSSVO so: » Es kann heute als feststehend gelten, daß der deutsche Gesetzgeber während des Weltkrieges den Mächten der Zersetzung nicht mit derjenigen Energie und Rücksichtslosigkeit entgegengetreten ist, die durch den Ernst der Stunde geboten war «. ${ }^{3}$ Der aus religiöser Überzeugung und Gewissensentscheidung gefaßte Entschluß, in Hitlers Krieg nicht mit der Waffe zu dienen, wurde damit den »Mächten der Zersetzung « zugerechnet.

Militärjuristen, auch die Richter des RKG, wandten den $\$ s$ KSSVO mit der Interpretation an, daß eine Zersetzungsabsicht des Täters nicht vorausgesetzt zu werden brauche, ja für den Untertatbestand $\$ s$ Abs. 1 Ziff. 3 sollte nach der herrschenden Auffassung das Merkmal der »Wehrkraftzersetzung " belanglos sein, weil andernfalls der »Wille des Gesetzgebers« verfehlt würde. ${ }^{4}$ Die Verweigerung aus Gewissensgründen stellte mithin selbst nach Auffassung der Judikatur und Kommentarliteratur keine Zersetzung der Wehrkraft dar: Zersetzungseigenschaft der Handlung war nicht conditio sine qua non einer Verurteilung zum Tode. Das RKG hat sich aus diesem Juristendilemma dadurch herauszumanövrieren versucht, daß es den nie bewiesenen Vorwurf der gefährlichen Werbekraft zu Hilfe nahm und damit die Notwendigkeit von Abschreckungsurteilen begründete.

Diese Praxis hat nach dem Krieg Unsicherheit ausgelöst. Admiral Bastian, Gerichtsherr des RKG vom September 1939 bis Oktober 1944, der zahlreiche derartige Abschreckungsurteile bestätigt und ihre Vollstreckung angeordnet hat, behauptet in seinen Lebenserinnerungen, ${ }^{5}$ Gewissenshandlungen seien so gut wie unantastbar gewesen, Abschreckungsurteile seien nicht gefällt worden.

Auch rückblickend enthüllt sich die Abschreckungsargumentation als Liebedienerei vor dem Willen des "Führers". Hitler hatte bei Kriegsbeginn verlauten lassen, er könne bei ernsthafter Wehrdienstverweigerung keine Gnade walten lassen. ${ }^{6}$ Diese Äußerung bezog sich zwar auf die Zeugen Jehovas, von denen bis Jahresende 1939 schon viele vom RKG zum Tode verurteilt worden waren, sie ist aber vom RKG auf alle religiösen Verweigerer bezogen worden. Dabei hätten durchaus Hitlers Richtlinien für die Strafzumessung bei Fahnenflucht Möglichkeiten für mildere Urteile entnommen werden können,? zumal bei Fahnenflucht schon eher an eine "Werbekraft" gedacht werden konnte. Aber selbst hier konnte u. a. von der Todesstrafe abgesehen werden, wenn für die Tat keine unehrenhaften Beweggründe bestimmend gewesen waren.

Bei der Verweigerung aus religiösen Gründen existierte eine »Werbekraft« so gut wie

2 Grundsatze der Reichskriegsanwaltschaft I I, in: Zeitschrift fur Wehrrecht (ZWR), 8(1942/43), S 284 ff. 3 Erech Schange: Milıarstrafgesetzbuch nebst Kriegssonderstrafreclutsverordnung, Berlin, 6. Aufl., 1944, S. 425 .

4 Ebd., S. 429 .

5 Max Bastian: Lebenserinnerungen I. 9. 1939-20. 10. 1945. Schreıbmaschinenmanuskrupt, 29. 12. 1956, BA-MA, Nachlaß Bastzan, N 192/1

6 So It. Marinerechtsabteilung v. 18. г2. 1939, OKM/AMA/MWehr/MR IV »Behandlung der Ernsten Brbelforscher ", BA-MA, RMD $4 / 486$.

7 Richtlınien v. I 4. 4. 1940. Sie sahen vor, daß von der Todesstrafe abgesehen werden konnte, wenn jugendliche Unuberlegtheit, schwierige hausliche Verhaltnısse oder andere nicht unehrenhafte Bcweggrunde fur den Tater hauptsachlich bestımmend waren 
nie. Erst recht nicht bei Soldaten der beiden großen Bekenntnisse, deren »Kirchenobere « den Dienst im Kriege bejahten und in derartigen Gewissenskonflikten keine Hilfe boten. Dies war auch der Fall bei Jagerstetters Bischof Fliesser (Linz), der durchaus mit eher taktischen Mitteln die Kirche vor Eingriffen der Partei zu bewahren versucht hat. Aber in der Frage des Gehorsams gegenüber der »Obrigkeit « vertrat er wie der Episkopat traditionelle Auffassungen auch im NS-Staat, zumal im Krieg: „Von Christus stammt das tapfere Wort, das zunächst ihm selbst, dann jedem Blutzeugen des Glaubens, aber auch dem christlichen Soldaten und stillen Opferseelen in Front und Heimat gilt, das heldische Wort: ,Verliere dein Leben, dann wirst Du es gewinnen. (Marc. 8, 35) ${ }^{8}$ Im persönlichen Gespräch vor Jägerstatters Einberufung will der dem Nationalsozialismus durchaus kritisch gegenüberstehende Bischof ihm »die Grundsätze der Moral über den Grad der Verantwortlichkeit des Bürgers und Privatmannes für die Taten der Obrigkeit « auseinandergesetzt haben. ${ }^{9}$ Fazit des Bischofs: Die Politik des Staates geht den Bürger nichts an. Er soll sich um seine Familie kümmern. Nur bei "außerordentlicher Einsprechung von oben« darf der Katholik sich gegen den Staat stellen.

Angesichts der Kriegs- und Kirchenpolitik des NS-Staates konnte der Bischof den Bauern Franz Jagerstätter nicht überzeugen. Der Bischof konnte sich der Wirkung der Persönlichkeit seines Gesprächspartners nicht entziehen. Noch im August I945 teilte er aber dem Pfarrer von St. Radegund mit, ${ }^{10}$ bei aller Achtung vor der subjektiven Haltung des Mannes könne er »nicht als objektiv gültiges Vorbild für seine Haltung zur Militärpflicht hingestellt werden«.

Jägerstetter ging es gar nicht um derartige Würdigungen. Er hat verzweifelt um Erkenntnis gerungen in der Frage, ob die Obrigkeitslehre der Kirche dem NS-Staat und seiner Politik zugute kommen dürfe. Seine Antwort war schließlich ein kompromißloses Nein. Nicht die Verbohrtheit eines Fanatikers, wie sie vom RKG den Bibelforschern zugeschrieben worden ist, hat hinter diesem Nein gestanden, sondern eine vom Gewissen geleitete klarsichtige moralische und politische Bewertung des NS-Systems. Diese Haltung wird belegt durch seine Erklärungen in der Hauptverhandlung vor dem 2. Senat des RKG an 6. Juli 1943. Ihm gegenüber saßen als Richter: Reichskriegsgerichtsrat Lueben als Verhandlungsleiter, General der Flieger Musshoff, Vizeadmiral Arps, Generalmajor Schreiber, Oberkriegsgerichtsrat Ranft. Sie hatten ihn bearbeitet, seine Einstellung zu ändern. Jágerstätter war sich völlig im klaren, welche Folgen er auf sich nahm. Er sagte diesen Richtern, daß er aufgrund seiner religiosen Einstellung den Wehrdienst mit der Waffe ablehne. Dann präzisierte er: Er würde gegen sein religiöses Gewissen handeln, wenn er für den nationalsozialistischen Staat kampfen würde. Er sei erst im Laufe des letzten Jahres zu der Überzeugung gelangt, daß er als gläubiger Katholik keinen Wehrdienst leisten dürfe. Er könne nicht gleichzeitig Nationalsozialist und Katholik sein. Den früheren Einberufungsbefehlen habe er Folge geleistet, weil er es damals noch für Sünde angesehen habe, den Befehlen des Staates nicht zu gehorchen. Aus christlicher Nächstenliebe war Jagerstätter bereit, Dienst als Sanitätssoldat zu leisten.

Mit diesem knappen, eindringlichen Aufriß seiner inneren Auseinandersetzung mit

8 Sylvesterpredigt Bischof Fliessers 1944 um Linzer Dom, Linzer Diozesan Blatt, Nr. 1, i7 f, Beılage, zıt. nach Erna Putz, Franz Jagerstetter... besser die Hande als der Wille gefesselt, Linz, 2 Aufl. 1978, S. I 60 Zur Milstarseelsorge der evangelıschen und katholıschen Kırche vgl. Manfred Messerschmidt, Aspekte der Milıtarseelsorgepolıtık in nationalsozialıstıscher Zeit, in: Militargeschichtlıche Mitteılungen (MGM) 3 (1968), S. 63-105, und zur Militarseelsorgepolitik $1 \mathrm{~m}$ Zweiten Weltkricg, in: MGM, s (1969), S. 37-85.

9 So wird Bischof Fhessers Auffassung in eincm Brief des Generalsckretars Vicbock vom Seelsorgeamt in Lınz an Pfarrer Arthofer v. 27. 2. 1946 wiedergegeben, zit. nach Putz (Fn. 8), S 171.

I0 Bischofliches Ordınariat Linz, Seclsorgeamt, an Pfarramt St. Radegund, i1. August 1945, zut nach Putz (Fn. 8), S. 149 
Kernfragen der christlichen Staatslehre im Angesicht des NS-Unrechtsstaates konnte er Richter dieses Staates nicht an ihre Verpflichtung, dem Recht zu dienen, gemahnen. "Recht« war ihnen das Geflecht von Konstruktionen, das sie selbst mit ihren Entscheidungen und mit der von Militär- und Ministerialjuristen formulierten KSSVO errichtet hatten. Der konsequente Weg Jägerstätters bis dahin ist zu verfolgen anhand eines von ihm vor dem Gespräch mit dem Linzer Bischof niedergeschriebenen Katalogs mit I I Fragen ${ }^{12}$ und der Aufzeichnungen im Tegeler Untersuchungsgefängnis sowie Aufzeichnungen aus den Jahren 1941, insbesondere die Politischen Aufzeichnungen, die er in einem Heft 2 zusammengefaßt hat. ${ }^{12}$ Hier überlegt er zum Anschluß: »Unsere Bischöfe werden vielleicht auch geglaubt haben, es dauert vielleicht nur eine kurze Zeit und dann zerfällt alles wieder und sie können durch ihre Nachgiebigkeit den Gläubigen viele Martern und Peinen ersparen, doch leider kam es halt anders.« ... »die Kirche Österreichs ließ sich gefangen nehmen«.

In Heft 3 wie im Fragenkatalog fragt er: Wie bringt man es fertig, zu gleicher Zeit Soldat Christi und Soldat der Nationalsozialistischen Revolution zu sein? Welcher Katholik getraut sich, diese Raubzüge, die Deutschland schon in mehreren Ländern unternommen hat und noch immer weiterführt, für einen gerechten und heiligen Krieg zu erklären?

Die RKG-Richter sprachen zwar nicht von einem heiligen Krieg, aber in zahlreichen Verweigerungs-Todesurteilen war die Rede vom Daseinskampf oder Freiheitskampf des deutschen Volkes, von volksfeindlicher Gesinnung. Der 2. Senat hat auch im Urteil gegen Jägerstätter davon gesprochen, er lehne es hartnäckig ab, »in Deutschlands schwerem Daseinskampf seine vaterländische Pflicht als Soldat zu erfüllen«. Eine derartige Haltung stellte sich für den Senat, wie er schon im Urteil vom 28. Juli 1942 gegen den Schützen Josef Kischka ${ }^{13}$ ausgeführt hatte, als »ehrlose Gesinnung « dar. Mit dieser Formel oder mit der Behauptung einer volksfeindlichen Gesinnung verbanden Richter des RKG ihr Verdikt der Ehrlosigkeit. Im Urteil gegen den zur Tatzeit noch nicht I8jährigen Kanonier Gustav Schnitger, ${ }^{14}$ dessen Eltern als Bibelforscher schon 1939 und 1940 in ein KZ verbracht worden waren, stellte der 4 . Senat am 15 . Juli 1942 lapidar fest: Er habe eine volks- und wehrfeindliche Gesinnung an den Tag gelegt. Eine milde Beurteilung sei ausgeschlossen. Der Gesichtspunkt der Abschreckung verlange die schwerste Bestrafung: "Deshalb mußte auf die Todesstrafe erkannt werden. Bei der ehrlosen Gesinnung, die die Tat des Angeklagten verrät, sind ihm gemäß $\$ 32$ RStGB die bürgerlichen Ehrenrechte auf Lebenszeit aberkannt worden.« Bei diesem Verständnis von Ehre und Ehrlosigkeit hatten religiöse Überzeugungen oder gar nichtkonforme politische Auffassungen nicht den Hauch einer Chance, als Gewissensentscheidungen gewürdigt zu werden.

Bei Jägerstätter stieß das Gericht auf einen nachdenklichen Menschen, der sowohl aus tiefer Religiosität als auch aus wachem politischen Urteil zum Ergebnis gelangt war, daß er als Christ in dem verbrecherischen Krieg keinen Waffendienst leisten dürfe. Damit stand für das Gericht fest: »Der Angeklagte hat sich durch sein Verhalten als ehrlos erwiesen . Und sie urteilten in der eingeübten Manier. ${ }^{15}$ Allein im ersten Kriegsjahr befanden sich unter den von der Wehrmachtjustiz gefällten s Is Todesur-

11 Putz (Fn. 8), S. 148 f., u. Erna Putz, Gefangnısbriefe und Aufzeıchnungen, Franz Jagerstatter verweıgert 1943 den Wehrdienst, Linz 1987 , S. 177 f.

12 Putz (Fn. II), S. 124 ff.

13 RKG, 2. Senat, Urtc1l II 83/42, BA-Zwischenarchiv, Dablwutz-Hoppegarten, Filmrolle M 108, Akte 40.

${ }_{1} 4 \mathrm{RKG}, 4$. Senat, Urteil IV 74/42, BA-Zwischenarchw Dablwutz-Hoppegarten, Filmrolle M 1008 , A kte $3 \mathrm{I}$

is Siehe zum gesamten Komplex Manfred Messerschmidt, Gewalt statt Recht. Die Verwergerer aus Gewissensgrunden vor dem Reichskriegsgericht, in: ders.: "W/as damals Recht war..." NS-Mulitar- und Strafjustiz im Vernichtungskrieg, hrsg. v Wolfram Wette, Essen 1996, S. 73-96 
teilen 112 Urteile des RKG gegen Wehrdienstverweigerer. Abschreckung gehörte zur Justizstrategie. Der Chef OKW, Keitel, teilte am IO. Juni 1940 dem Admiral Bastzan mit, die scharfe Praxis finde Hitlers Zustimmung. Keitel bemerkte, das RKG habe sich "um die Erhaltung der Wehrkraft des Volkes ein Verdienst erworben und zugleich bewahrend und verhütend - menschlich viel Gutes getan. ${ }^{16}$ Menschlich viel Gutes: Die Urteilsgründe gegen den Baupionier Hermann Abke ${ }^{i 7}$ waren z. B. auf einer halben Schreibmaschinenseite abgehandelt. Viele Urteile sind so wortlautidentisch, daß sie einer Schablone zu entsprechen scheinen. ${ }^{8}$

Kann eine solche Verfahrensweise rechtsstaatlich genannt werden? Allein die Funktion des Gerichtsherrn spricht dagegen. In den »Erläuterungen zur Verordnung über das militärische Strafverfahren im Kriege und bei besonderem Einsatz " vom 17. August $1938^{19}$ war bestimmt: "Das Schwergewicht des militärischen Strafverfahrens ruht beim Gerichtsherrn. Er ist die alles beherrschende Persönlichkeit und trägt die Verantwortung dafür, daß mit der nötigen Schärfe und Beschleunigung durchgegriffen wird. Admiral Bastian hat in seinen Lebenserinnerungen aufgezeichnet, wie er diese gerichtsherrlichen Aufgaben angepackt hat. Nach Vorlage der Anklageschrift erließ er zusammen mit dem Oberreichskriegsanwalt (ORKA) oder dessen Beauftragten die Anklageverfügung. Vor der Hauptverhandlung erörterte er mit dem ORKA oder dem Sachbearbeiter, welche Strafe »auszuwerfen « sei. ${ }^{20}$ Die Richter des erkennenden Senats hatten auf diese Weise vor Augen, welche Strafe der für die Bestätigung des Urteils zuständige Gerichtsherr für geboten hielt. Bastzan drängte wiederholt auf zügige Verfahrensweisen. Einen Richter, der abweichende Voten hinterlegte und sich für die Beiziehung weiterer Beweismittel im Interesse der Angeklagten eingesetzt hatte, schlug er zur vorzeitigen Versetzung in den Ruhestand vor. Auch in der Bestätigungsfrage schaltete er den ORKA oder andere Beteiligte ein. So ist es ihm nach eigener Bekundung immer schwerer gefallen, Urteile aufzuheben. Er spricht yom Respekt vor der »richtigen Einstellung des Richtertums zu seiner erhabenen Aufgabe. ${ }^{21}$ Dies war die Art und Weise, wie er die Rechtsprechung des RKG dahin dirigierte, »die Schlagfertigkeit der Wehrmacht zu stärken und sich an die Kriegslage anzupassen. ${ }^{22}$

Todesurteile im Schnellverfahren gegen Verweigerer aus religiösen Gründen konnten schwerlich die Schlagfertigkeit der Wehrmacht stärken. Im Ersten Weltkrieg hat die Armee derartige Urteile nicht für erforderlich gehalten. Die westlichen Alliierten haben wegen conscientious objection keine Todesurteile verhängt. Diese blieben der Wehrmacht des NS-Staates und ihren Richtern vorbehalten. Die Urteile waren politische Resultate der NS-Gemeinschaftsideologie.

So ist das Urteil gegen Franz Jagerstatter einzuordnen. Sein Schicksal berührte sich mit dem des Pallotinerpaters Franz Dionysius Reinisch, der vom 3. Senat des RKG am 7. Juli 1942 zum Tode verurteilt worden ist. Bastian bestätigte das Urteil am I. August und ordnete die Vollstreckung an. ${ }^{23}$ Der Gefängnisgeistliche Heinrich Kreutzberg hat Jagerstätter nach seiner Verurteilung von der Haltung des Reinisch erzählt.

16 Wiedergegeben be Norbert Haase, Das Reichskrıegsgericht und der Widerstand gegen die nationalsozıalıstısche Herrschaft (Katalog zur Sondcrausstellung der Gedenkstatte "Deutscher Wıderstand “), Berlın 1993, S. 51 .

$17 \mathrm{RKG}$, x. Senat, Urtesl 45/44, BA-Zwischenarchiv Dablwiz-Hoppegarten, Filmrolle M 1004, Akte 34 .

I 8 Die beiden am 2. Dezember 1943 vom 4. Scnat (Verhandlungsletter RKG-Rat Dr. Lattmann) gegen Kurt We u. 83/43, Zwischenarchiv Dahlwatz-Hoppegarten, Filmrolle M roos, Akten A65 u. 66.

19 Anhang zur HDv. 3/1 3, abgedruckt be Rudolf Absolon, Das Wehrmachtstrafrecht im Zweiten Weltkrieg. Sammlung der grundlegenden Gesetze, Verordnungen und Erlasse, Kornelımunstet 1958, S. 179-189.

20 Bastian, Lebenserınnerungen (Fn. 3), S. 26

21 Ebd., S. 38

22 So verlangte er es mit Dienstlichen Richtlınien v. I4. August 1944, zit. be1 Haase (Fn. 16), S. 44 f

23 Urteil RKG, 3. Senat, III 70/42, BA-Zwischenarchiv Dablwitz-Hoppegarten, Filmrolle M roo8, Akte 9. 
Das Beispiel hat Jägerstatter zutiefst beeindruckt. ${ }^{24}$ Reinisch hatte offen erklärt, die Liebe zum deutschen Volk und zu seiner Heimat Tirol zwinge ihn, gegen den Nationalbolschewismus zu kämpfen. Er stand wie Jägerstätter auch im kirchlichen Raum einsam da. Eine ähnliche Verbindung von Gewissensskrupeln und politischer Hellsichtigkeit wie bei Jagerstatter charakterisiert seine Handlungsweise. Weil er zum Subdiakon geweiht war, konnte er nach den Bestimmungen der Wehrmacht nur zum Sanitätsdienst einberufen werden, den er konsequent abgelehnt hat. Insofern war seine Position »rechtlich" anders gelagert als die Jigerstatters, der ohne Ausnahme dienstpflichtig war. Seine Bereitschaft, lediglich Sanitätsdienst leisten zu wollen, haben die Richter als teilweise Wehrdienstentziehung ausgelegt und damit $\mathbb{S}$ Abs. 1 Ziff. 3 KSSVO herangezogen. Es machte keinen Unterschied, daß bei Reinisch der scharfe Senatspräsident Schmauser und bei Jagerstatter der weniger hartnäckige RKG-Rat Lueben Regie führte, gleichgültig auch, ob die Angeklagten vor dem 3. oder 2. Senat zu erscheinen hatten. Für diese Richter waren sie chrlos. Der 2. Senat unter Lueben hat dies bei Jagerstatter sogar noch deutlicher zum Ausdruck gebracht, und Bastian bestätigte mit seiner Unterschrift diese »Ehrauffassung ", wovon in seinen Lebenserinnerungen allerdings nicht die Rede ist.

Die Urteile gegen Jagerstatter und Reinisch sind nun durch Beschluß des Landgerichts Berlin und durch die bayerische Justiz aufgehoben worden. Am 3. Juni 1997 hat das Landgericht für Strafsachen Wien ein weiteres Urteil gegen einen Verweigerer aus religiösen Gründen aufgehoben, nämlich das Todesurteil des RKG vom 22. Januar 1943 gegen Anton Uran, Zeuge Jehovas, der am Tage der Urteilsverkündung noch nicht 23 Jahre alt war. Das Landgericht hat festgestellt, daß Verurteilungen gemäß $\$ 5$ KSSVO aufgrund des österreichischen Gesetzes vom 3. Juli 1.945 über die Aufhebung von Strafurteilen und die Einstellung von Strafverfahren aufzuheben sind und hat entsprechend entschieden. Dieses Urteil ist eindeutig. Obwohl das Berliner NS-StrWG ebenfalls vorsieht, daß Urteile, die auf vom Alliierten Kontrollrat aufgehobenen NS-Vorschriften beruhen, aufzuheben sind und obwohl die amtliche Begründung zu $\$ 1$ dieses Gesetzes die möglichst umfassende Wiedergutmachung des nationalsozialistischen Unrechts in der Strafrechtspflege als Ziel des Gesetzes angibt und somit eine weite Auslegung geboten ist, hat das Kammergericht mit Beschluß vom 14. November $1996^{25}$ den Standpunkt vertreten, daß in solchen Fällen "eine widerlegliche Vermutung « indiziere, daß die Voraussetzungen des $\mathbb{~ I ~ A b s . ~ I ~}$ NS-StrWG vorliegen. Dieser sehr zurückhaltenden Interpretation des Gesetzes hat das Landgericht sich angeschlossen. Sein Beschluß kann nicht als kompromißlose Lösung bezeichnet werden. Möglicherweise hat die Kammer deswegen auch ausgeführt, eine Wehrdienstverweigerung habe bei Jägerstätter tatbestandlich nicht vorgelegen. Diese Annahme verharmlost den Willen des NS-Gesetzgebers, der bewußt auch die Ablehnung bestimmter Anforderungen des Wehrdienstes als Verweigerung bestraft sehen wollte.

Was besonders nachdenklich stimmen muß, ist das Fehlen jeder Andeutung des Widerstandscharakters der Haltung Jägerstätters. Er wie Reinisch haben offen zum Ausdruck gebracht, daß sie das NS-System und seine Politik ablehnten. Dies insbesondere hat ihnen den Vorwurf der Ehrlosigkeit eingetragen von Richtern, die dieses System bis zum Zusammenbruch gestützt und stabilisiert haben. Der Einzelne hat die Moral des staatlichen Willens nicht zu beurteilen. So sahen es der Staat, die Partei, die Justiz und die Kirchen. Noch zwanzig Jahre später befand der Bundesgerichtshof, kein Staat könne dem Einzelnen gestatten, über die Rechtmaßigkeit eines Krieges zu

24 Hierzu Georg Bergmann, Franz Jagerstatte) Ein Lcben vom Gewissen entschieden, Stem a. Rhen, 2. Aufl. 1988, S. 311 f.

25 NJW $1997, \mathrm{~S} 953,956$ 
befinden. Auffassungen von Verweigerern wie des reformierten Theologen Dr. Wilbelm Schümer paßten in das Obrigkeitsverständnis auch der evangelischen Kirche nicht hinein. Für Schumer stand fest, daß der Christ die "Vergöttlichung des Führers “ und den totalen Staat ablehnen müsse. Vergebens suchte Gefängnispfarrer Harald Poelchau Bischof Mabrarens (Hannover) auf, um seine Stimme für Hermann Stöhrzu gewinnen, einen ebenso konsequenten protestantischen Verweigerer. Poelchaus Resümee: »Das Versagen der offiziellen Kirche ... war für die Christenheit beschämend ${ }^{26}{ }^{26}$

Für protestantische und katholische Verweigerer hat die Einstellung der Kirchen den Effekt einer politisch-geistigen Isolierung gehabt, wie sie bei Zeugen Jehovas in dieser Form nicht gegeben war, weil sie sich in ihrer Glaubensgemeinschaft verstanden fühlen durften. Mit dieser Isolierung sind die Angehörigen der katholischen und protestantischen Verweigerer auch nach dem Krieg konfrontiert gewesen. Die Justiz verlängerte die Wirkungen der Isolierung. Erst neuere Entscheidungen höchstrichterlicher Rechtsprechung werden dem widerständigen Verhalten der Verweigerer gerecht. Vorausgegangen ist das Bundessozialgericht mit seinem Urteil vom 1 I. September $199 \mathrm{I},{ }^{27}$ wo es heißt, der Abschreckungszweck könne nicht von der »konkreten politischen Situation, in der dieser Krieg geführt worden ist, losgelöst werden ... die Todesstrafe wurde um der Kriegführung willen so zwangsläufig verhängt wie in den Urteilen des Volksgerichtshofs. « Die Annahme, es habe in Militärstrafsachen ein rechtsstaatliches Verfahren gegeben, so das BSG, begegne erheblichen Bedenken. Die Vorstellung, Wehrdienst sei Ehrendienst, habe eingeschlossen, daß Soldaten, die nicht gehorsam »Ehrendienst « leisteten, sterben mußten. Das Gericht spricht von der Vermutung der $»$ offensichtlichen Ungerechtigkeit von Todesurteilen«. Der s. Senat des BGH hat sich in seiner Entscheidung vom 16. November 1995 eindeutig von der früheren Rechtsprechung des Gerichts zur Frage der Rechtsbeugung durch NS-Richter distanziert und spricht nun von einer "Perversion der Rechtsordnung", wie sie "schlimmer kaum vorzustellen« gewesen sei. ${ }^{28}$ Der Bundesgerichtshof erklärt Zehntausende von Todesurteilen fur unwirksam, weil die »Kriegsrichter « keine unabhängigen Richter waren, sie haben »dazu beigetragen, den völkerrechtswidrigen Krieg zu führen. «"2)

Die 8. Synode der Evangelischen Kirche in Deutschland hat auf ihrer 7. Tagung am 6. November 1996 festgestellt, der Zweite Weltkrieg sei ein von NS-Deutschland verschuldetes Verbrechen gewesen. Auch die Kirche müsse dies erkennen: "Wer sich weigert, sich an einem Verbrechen zu beteiligen, verdient Respekt." Eine solche Weigerung könne nicht strafwürdig sein.

Franz Jagerstatter hat sich geweigert. Er stand von Anfang an auf der richtigen Seite. Es ist nur viel zu spät erkannt worden.

26 Harald Poelchau, Die Ordnung der Bedrangten, Munchen ig6s, S 7s f

27 BSG, Az:: 9 a RV $11 / 90$

28 NJW 1996, S. 857

29 S. zu diesem Urteil dic Besprcchung von RA Dr. Otto Grtschneder; NJW 1996, S. 1239 ff 